\title{
Innervation imaging to guide ventricular arrhythmia ablation
}

\author{
Andreas A. Giannopoulos, MD, PhD, ${ }^{\mathrm{a}}$ and Aju P. Pazhenkottil, $\mathrm{MD}^{\mathrm{a}}$ \\ a Cardiac Imaging, Department of Cardiology and Nuclear Medicine, University Hospital Zurich, \\ Zurich, Switzerland
}

Received Jan 24, 2019; accepted Jan 24, 2019

doi: $10.1007 / \mathrm{s} 12350-019-01632-\mathrm{z}$

\section{See related article, pp. 175-183}

Catheter ablation is a modern treatment approach for patients with ventricular arrhythmias in the case that antiarrhythmic medications are either not effective, not well tolerated, or even refused by the patient. ${ }^{1}$ In cases of ventricular arrhythmias post myocardial infarction, scar formation pertaining areas of myocardial fibrosis intermingled with viable myocytes represents the most common substrate. ${ }^{2}$ Slow conduction within the scarred subendocardial surface of the left ventricle denotes the most common underlying mechanism of ventricular tachyarrhythmias. ${ }^{3}$

Ventricular tachycardia ablation procedures are commonly guided by delineation (mapping) of the substrate, whereby regions of scared tissue and potential reentry circuit are outlined in electroanatomic maps based on electrocardiographic and pacing characteristics assessed during hemodynamically stable sinus or paced rhythm. Despite technological progress and improved experience of operators, success rate remains on the lower side, with a recurrence rate of up to $47 \%$ during the first 6 months. ${ }^{4}$ Several substrate-based ablation techniques are used, of which the elimination of local abnormal ventricular activities is one of the most prominent that is constantly gaining ground and that has been associated with long-term freedom of ventricular tachyarrhythmia relapse. ${ }^{5}$

Reprint requests: Aju P. Pazhenkottil, MD, Cardiac Imaging, Department of Cardiology and Nuclear Medicine, University Hospital Zurich, Raemistrasse 100, 8091 Zurich, Switzerland; aju.pazhenkottil@usz.ch

J Nucl Cardiol 2021;28:184-6.

$1071-3581 / \$ 34.00$

Copyright (C) 2019 American Society of Nuclear Cardiology.
Pre- and peri-procedural imaging for substratebased ablation has so far mainly been implemented by using cardiac magnetic resonance as well as cardiac computed tomography aiming to accurately segment the myocardium and provide detailed myocardial tissue characterization. $^{6-8}$ The former provides adequate image resolution for detection and localization of non-transmural scar areas including tissue characterization, whereas the latter can be performed in patients with implantable cardioverter defibrillators, which represent a significant number of patients with ventricular tachyarrhythmias post myocardial infarction.

Such anatomical planning in advanced catheter ablation enables patient-specific mapping and guidance during the intervention. Still, critical physiological information, pertinent to sympathetic denervation in scarred areas, cannot be conveyed with cross-sectional imaging. In fact, sympathetic cardiac innervation plays an important role in ventricular arrhythmogenesis and may act as a trigger and substrate modulator of the ventricular tachyarrhythmia substrate. Sympathetic nervous system degeneration and necrosis of sympathetic fibers after myocardial infarction are usually extending to an area greater than that of myocardial necrosis. ${ }^{9}$ Myocardial ventricle tissue with preserved viability but with sympathetic denervation is particularly sensitive to sympathetic stimulation and could thereby predispose to tachyarrhythmias. ${ }^{10} \quad{ }^{123}$ I-labeled metaiodobenzylguanidine $\left({ }^{123} \mathrm{I}-\mathrm{MIBG}\right)$ cardiac nuclear imaging can assess cardiac adrenergic nerve function and has the potential to identify larger zones of susceptibility to ventricular tachyarrhythmias.

In the current issue of the Journal of Nuclear Cardiology, Gimelli and colleagues ${ }^{11}$ investigated for the first time the correlation between regional sympathetic innervation as assessed with ${ }^{123}$ I-MIBG and perfusion assessed by ${ }^{99 \mathrm{~m}}$ Tc-tetrofosmin in ischemic cardiac disease patients that underwent ablation of local abnormal ventricular activities. Sixteen post myocardial 
infarction patients underwent a combined evaluation with dual-tracer scintigraphy at baseline and at 1 month following the ablation procedure. The summed perfusion rest score and the summed ${ }^{123}$ I-MIBG score were calculated by adding the segmental scores of the perfusion and innervation, respectively. The presence of perfusion-innervation mismatch was defined as a difference of more than $25 \%$ of the relative uptake of $99 \mathrm{mTc}$-tetrofosmin minus that of ${ }^{123} \mathrm{I}-\mathrm{MIBG}$. An elevated regional burden of perfusion-innervation mismatch was shown to correlate with arrhythmic substrate. On follow-up scan, ablated segments showed a significant reduction of ${ }^{99 \mathrm{~m}}$ Tc-tetrofosmin uptake resulting in a significant reduction in the extent of perfusion-innervation mismatch. Importantly, reduction of the mismatch post ablation was associated with improved clinical outcome at one-year follow-up with a significant reduction of the arrhythmic burden.

The authors should be congratulated for elaborating on this novel, pathophysiological rational approach on improving the planning of ventricular arrhythmia ablation and adding potentially a predictive tool on the recurrence of ventricular arrhythmias in the future. These results come into a compelling background of data and are consistent with existing knowledge on the still viable but denervated left ventricular myocardial tissue and the associated jeopardy of ventricular arrhythmias. ${ }^{12,13}$ Nevertheless, as for every study, there are certain limitations that deserve closer attention. Notably, this pilot study included only a small number of patients and focused on a particular ablation strategy. Furthermore, the combination of anatomical information coming from other imaging modalities such as computed tomography or magnetic resonance imaging could potentially improve the pre-intervention planning. Finally, manually co-registration of the scintigraphy and electroanatomical mapping datasets might have caused minimal misregistration flaws.

Certainly, prospective randomized studies comparing hybrid anatomical and functional imaging-guided ventricular tachyarrhythmia ablation to standard ablation in the setting of different diseases are necessary to determine whether this promising technology can improve short- and long-term outcomes. In the era of personalized medicine, generalized, blind approaches are expected to give their place to patient-specific treatment supported by clinical evidence that would have an impact on the long-term prognosis. Guiding electrophysiological interventions in patients with ventricular tachyarrhythmias post myocardial infarction with proper pre-intervention imaging, using additional functional data instead of anatomical information alone, represents a step down the appropriate road.

\section{Disclosure}

The University Hospital Zurich holds a research contract with GE Healthcare. A.P.P. and A.A.G. report that they have no relationships relevant to the contents of this editorial to disclose.

\section{References}

1. Al-Khatib SM, Stevenson WG, Ackerman MJ, Bryant WJ, Callans DJ, Curtis AB, et al. 2017 AHA/ACC/HRS guideline for management of patients with ventricular arrhythmias and the prevention of sudden cardiac death: A Report of the American College of Cardiology/American Heart Association Task Force on Clinical Practice Guidelines and the Heart Rhythm Society. Heart Rhythm 2018;15:e73-189.

2. de Bakker JM, van Capelle FJ, Janse MJ, Tasseron S, Vermeulen JT, de Jonge $\mathrm{N}$, et al. Slow conduction in the infarcted human heart. 'Zigzag' course of activation. Circulation 1993;88:915-26.

3. Piers SR, Zeppenfeld K. Imaging-guided Ventricular Tachycardia Ablation. Arrhythmia Electrophysiol Rev 2013;2:128-34.

4. Stevenson WG, Wilber DJ, Natale A, Jackman WM, Marchlinski FE, Talbert T, et al. Irrigated Radiofrequency Catheter Ablation Guided by Electroanatomic Mapping for Recurrent Ventricular Tachycardia After Myocardial Infarction. Circulation 2008;118:2773-82.

5. Jaïs P, Maury P, Khairy P, Sacher F, Nault I, Komatsu Y, et al. Elimination of Local Abnormal Ventricular Activities. Circulation 2012;125:2184-96

6. Sasaki T, Miller CF, Hansford R, Yang J, Caffo BS, Zviman MM, et al. Myocardial structural associations with local electrograms: a study of postinfarct ventricular tachycardia pathophysiology and magnetic resonance-based noninvasive mapping. Circ Arrhythmia Electrophysiol 2012;5:1081-90.

7. Dickfeld T, Schuleri KH. "Almost There": The Princess and the Frog Song*. JACC 2016;9:833-5.

8. Esposito A, Palmisano A, Antunes S, Maccabelli G, Colantoni C, Rancoita PMV, et al. Cardiac CT With Delayed Enhancement in the Characterization of Ventricular Tachycardia Structural Substrate: Relationship Between CT-Segmented Scar and Electro-Anatomic Mapping. JACC Cardiovasc Imaging 2016;9:822-32.

9. Gimelli A, Masci PG, Liga R, Grigoratos C, Pasanisi EM, Lombardi $\mathrm{M}$, et al. Regional heterogeneity in cardiac sympathetic innervation in acute myocardial infarction: relationship with myocardial oedema on magnetic resonance. Eur J Nucl Med Mol Imaging 2014;41:1692-4.

10. Kammerling JJ, Green FJ, Watanabe AM, Inoue H, Barber MJ, Henry DP, et al. Denervation supersensitivity of refractoriness in noninfarcted areas apical to transmural myocardial infarction. Circulation 1987;76:383-93.

11. Gimelli A, Menichetti F, Soldati E, Liga R, Scelza N, Zucchelli G, et al. Predictors of ventricular ablation's success: viability, innervation or mismatch? J Nucl Cardiol 2019. https://doi.org/10. 1007/s12350-018-01575-x

12. Klein T, Abdulghani M, Smith M, Huang R, Asoglu R, Remo BF, et al. Three-dimensional 123I-meta-iodobenzylguanidine cardiac innervation maps to assess substrate and successful ablation sites for ventricular tachycardia: feasibility study for a novel paradigm 
of innervation imaging. Circ Arrhythm Electrophysiol 2015;8:58391.

13. Gimelli A, Menichetti F, Soldati E, Liga R, Vannozzi A, Marzullo $\mathrm{P}$, et al. Relationships between cardiac innervation/perfusion imbalance and ventricular arrhythmias: impact on invasive electrophysiological parameters and ablation procedures. Eur J Nucl Med Mol Imaging 2016;43:2383-91.

Publisher's Note Springer Nature remains neutral with regard to jurisdictional claims in published maps and institutional affiliations. 